Revista de Matemática: Teoría y Aplicaciones 2008 15(1) : 49-70

CIMPA - UCR ISSN: 1409-2433

\title{
LA ECUACIÓN DE NAVIER-STOKES Y MULTIFRACTALES
}

\author{
José Roberto Mercado Escalante*
}

Recibido/Received: 22 Feb 2006; Versión revisada/Revised version: 11 Aug 2007; Aceptado/Accepted: 31 Oct 2007

\begin{abstract}
Resumen
No existe, en la actualidad, un teorema general sobre la existencia y la unicidad de las soluciones de la ecuación de Navier-Stokes, la cual describe el flujo de un fluido viscoso e incompresible. Éste es un problema abierto a nivel internacional, llamado el Problema del Premio del Milenio, por el cual el Instituto Clay de Francia está ofreciendo 1 millón de dólares, desde mayo de 2000.

Nuestro propósito, con el presente artículo, es presentar una revisión breve sobre los aspectos más importantes de la evolución y estado actual del problema.

Nuestro aporte es la descripción analítica de la turbulencia, completamente desarrollada, a través de las tasas de la resolución y de los rasgos de procesos multifractal, como una colección de procesos de Cantor generalizados. Presentamos cuatro modelos para la distribución de las variaciones de la velocidad; el primero lo basamos en los tiempos de vida y funciones de riesgo para la interacción entre los vórtices y su posterior fragmentación en vórtices cada vez más pequeños y más numerosos; el segundo, se basa en las pruebas de Bernoulli potenciadas, y encontramos el número de rasgos, el espectro y la función de estructura. Encontramos la relación de los parámetros de forma con la dimensión caja del máximo del espectro; como también, con las dimensiones locales. Y, describimos cuantitativamente el árbol asociado.

Las tasas mencionadas nos sirven de soporte, no sólo, para la descripción de un modelo tridimensional de turbulencia intermitente, que generaliza el resultado paradigmático de Kolmogorov; sino además, la energía transferida en cada etapa del proceso de fractalización; como también, el número de los exponentes característicos, el cual produce una cota superior para la dimensión de Hausdorff del conjunto de singularidades de las soluciones.
\end{abstract}

Palabras clave: Navier-Stokes, turbulencia, intermitencia, multifractales, gradientes de velocidad.

*Instituto Mexicano de Tecnología del Agua, IMTA, Paseo Cuauhnáhuac 8532, Progreso, Jiutepec, Mor., C. P. 62550, México. Fax: +\{52\} 77732936 00. E-Mail: rmercado@tlaloc.imta.mx 


\begin{abstract}
There is currently no general theorem on the existence and unicity of solutions to the Navier-Stokes equation, which describes the flow of a viscous and incompressible fluid. This is an open problem at the international level, known as the millennium prize problem, for which the Clay Institute of France is offering one million dollars since may 2000.

The purpose of this article is to present a brief revision of the most important aspects of the evolution and current status of the problem. Our contribution is the analytical description of turbulence, fully developed, through the resolution rates and the features of multifractal processes, as a collection of generalized Cantor processes. We present four models for the distribution of velocity variations. The first one is based on the life times and risk functions for the interaction between the vortices and their later fragmentation in ever smaller and more numerous vortices. The second one is based on potentiated Bernoulli tests, and we found the number of features, the spectrum, and the structure function. We found the relationship of the shape parameters with the box dimension of the maximum spectrum as well as with the local dimensions and we described qualitatively the associated tree.

The above-mentioned rates serve as support, not only for the description of a three-dimensional model of intermittent turbulence that generalizes the Kolmogorov paradigmatic result, but also for the energy transferred in each stage of the fractalization process, and also for the number of characteristic exponents, which produces a higher level for Hausdorff's dimension of the set of singularities of the solution.
\end{abstract}

Keywords: Navier-Stokes, turbulence, intermittency, multifractals, velocity gradients.

Mathematics Subject Classification: 76F55, 60G18, 60G70.

\title{
1 Introducción
}

Como es ampliamente conocido, la ecuación de Navier-Stokes surge de aplicar la segunda ley de Newton, o ley de la fuerza, a un elemento de masa sometido a las interacciones de las fuerzas de esfuerzos, en la que participa la presión; y a las fuerzas de cuerpo, entre las que se encuentran la fricción y la gravedad; junto con la condición de la conservación de la masa, que debido a la característica de la incompresibilidad del fluido, se convierte en la ausencia de divergencia; y en donde, además, se debe agregar la condición inicial para la velocidad del fluido.

Se sabe que, en tres dimensiones, cuando la velocidad inicial es condicionalmente pequeña, existen una función regular para la presión, y funciones regulares para las tres componentes de la velocidad que satisfacen las condiciones requeridas, especificadas por la ecuación diferencial, la conservación de la masa, la condición inicial, y la energía cinética acotada; siendo las componentes de la velocidad y la presión regulares de todo orden, (1934). Estos resultados sirven de base para construir los distintos métodos numéricos que permiten obtener las aproximaciones a las soluciones de la ecuación de Navier-Stokes.

Sin embargo, cuando no se somete la condición inicial a aquella restricción de pequeñez, puede surgir un instante de tiempo para el cual la solución deja de tener la energía cinética acotada y se dice que la solución explota en un tiempo finito. 
Como es usual en matemáticas, es apropiado crear un concepto débil, que permita hacer el análisis de las condiciones en forma más sencilla, para luego obtener un camino de tránsito del concepto débil al fuerte. Con esta visión, el problema ha sido abordado desde el ángulo de las soluciones débiles. Se sabe que las ecuaciones de conservación generan soluciones discontinuas como los del problema de Riemann, por lo cual E. Hopf introdujo las soluciones débiles, y Peter Lax prosiguió su desarrollo ulterior, [11], [12]. Para definir las soluciones débiles se concibe a la ecuación diferencial como una funcional, compuesta de términos que también son funcionales. Se las evalúa en las funciones de prueba; la integración por partes de Leibniz, o el teorema de Green, traspasan las derivadas parciales desde las variables de campo a las funciones de prueba, las que se asumen diferenciables de todos los ordenes. Se dice entonces que se cuenta con la formulación débil, o variacional, de la ecuación diferencial.

Una estrategia global de la solución del problema abierto, mencionado en el resumen, consiste en construir una solución débil y luego la tarea se concentra en el intento de demostrar que cada solución débil de la ecuación de Navier-Stokes, es regular.

Se demuestra la existencia de soluciones débiles que cumplen con las condiciones requeridas; lo que puede alcanzarse, al estructurar un proceso de Galerkin, que conduce a un sistema de ecuaciones diferenciales ordinarias, pero aún cuando, subsiste la presencia de la característica no lineal que induce la forma trilineal del término convectivo, la dificultad se supera buscando las condiciones para extraer una subsucesión que permanezca en un dominio acotado fijo y que posibilite transformar la convergencia débil en fuerte, hacía la solución buscada, apelando a un criterio de compacidad. Para el caso bidimensional el resultado se refuerza aún más, alcanzándose la unicidad de la solución débil, (1959).

No se conoce un resultado sobre la unicidad de las soluciones débiles; en el mismo contexto espacial en donde se garantiza su existencia; pero sí se tiene esta unicidad, aunque para un ámbito espacial más reducido, (1969). En general si se exige un incremento en la regularidad de la solución, puede mejorarse el resultado sobre la unicidad.

En particular, para las ecuaciones de Euler, es decir, sin viscosidad, está demostrado que no se cuenta con la unicidad; que por lo demás, es lo frecuente en el marco de las soluciones débiles; por ejemplo, para una ecuación de conservación, se obtiene la unicidad para las soluciones débiles, introduciendo una condición adicional que se sustenta en su significado físico y que se conoce como condición de entropía, [22].

En nuestro caso, las razones físicas se expresan señalando que las derivadas de la condición inicial deben satisfacer un crecimiento condicionado, en tanto que la energía cinética acumulada se mantenga acotada, a lo largo de todos los instantes del proceso evolutivo, (ecs. (2), (3)).

Scheffer, que inicia el estudio de la teoría de la regularidad parcial, se mueve en otra dirección respecto de esta misma regularidad, y establece en 1977, que las soluciones de la ecuación de Navier-Stokes son continuas excepto en un conjunto cerrado, el conjunto singular, cuya dimensión de Hausdorff no excede el valor 2. Caffarelli et al. (1982), y luego Lin (1998), refuerzan y amplían ese resultado obteniendo que la dimensión de Hausdorff parabólica del conjunto singular no supera el valor de 5/3. Pero este resultado aún no cancela la estrategia planteada de demostrar que cada solución débil es regular, [21].

Con abundante frecuencia los flujos en la naturaleza y la industria son turbulentos, 
[18], por lo que surge la necesidad de considerar la ecuación diferencial de la evolución de los vórtices. Ésta se desarrolla desde las valiosas observaciones, anotaciones y dibujos de Leonardo Da Vinci acerca de los vórtices, que figuran en la recopilación conocida como el Arconati, [13]; pasando por la propuesta de Boussinesq de 1877, y de Reynolds de 1894, [5]; hasta los resultados de Kolmogórov sobre el espectro de energía de la turbulencia, de 1941; quien encontró el valor de la potencia 5/3 a través del análisis dimensional, y que en la actualidad se obtiene por el teorema Pi de Buckingham, y cuenta con amplia verificación tanto experimental como numérica, [18], [4].

En este artículo, primero destacamos la ecuación de Navier-Stokes junto con sus condiciones limitantes, (ecs (1), (2), (3)), y enunciamos el problema abierto citado. Al final de la sección, llegamos a la parte hermítica del operador de evolución, en donde se destaca la tasa de disipación de la energía por unidad de masa. En la sección (3), revisamos la construcción de medidas multifractales. La medida micro y su soporte. Vemos los cambios de escala en la ecuación de Navier-Stokes. Proponemos los coeficientes de la ecuación Fokker-Planck para obtener la densidad de probabilidad y su interpretación, lo que nos produce el número de rasgos, de lo cual se hallan el espectro, las dimensiones locales y la función de estructura. Se obtiene la relación entre el parámetro de forma y la dimensión del soporte de la medida. Aproximamos la densidad de la distribución multifractal por una gaussiana y una Lévy. Encontramos el modelo multifractal basado en los procesos potenciados de Bernoulli y en el modelo paramagnético de Curie. Describimos el árbol o cascada energética para hallar el espectro de energía en su expresión multifractal, por lo que emerge la distribución Beta y sus dos parámetros de forma dependientes de los índices de dimensiones y Lévy; y su transformada de Laplace, con su forma acampanada. El sistema de Pearson nos presenta otra forma de superar la distribución gaussiana y modelar las pequeñas escalas; con lo que obtenemos nuevamente la distribución Beta, con parámetros de forma que se determinan de la asimetría y la curtosis, los que a su vez dependen de los dos índices: dimensiones y Lévy. Las distribuciones de los gradientes de velocidad las podemos determinar partiendo del soporte de la gaussiana y su cercanía con la multifractal. En las últimas secciones, enunciamos las conclusiones y señalamos las referencias básicas citadas.

\section{Parte I: Problema abierto y modelo de extremos}

\section{Ecuaciones de movimiento}

La ecuación de Navier-Stokes es una ecuación de evolución que describe la velocidad de flujo de un fluido viscoso e incompresible, en un espacio de divergencia nula que expresa la conservación de la masa bajo la condición de incompresibilidad del fluido; y que parte de un estado inicial. Se denota por: $\nu>0$ la viscosidad cinemática, $p$ la presión, $\rho$ la densidad del fluido, $\Delta$ el operador de Laplace, $\nabla$ el operador gradiente, $x$ el vector espacial de posiciones y $t$ el tiempo; la ecuación es:

$$
\frac{\partial}{\partial t} \mathbf{u}+(\mathbf{u} \cdot \nabla) \mathbf{u}=\boldsymbol{\nu} \boldsymbol{\Delta} \mathbf{u}-g r a d \frac{p}{\rho}+\mathbf{f}, \quad \nabla \cdot \mathbf{u}=0, \quad \mathbf{u}(x, 0)=\mathbf{u}_{0}(x)
$$


A la condición inicial y a las fuerzas se agregan la condición de su comportamiento en el horizonte lejano, $x \rightarrow \infty$; siendo arbitrarios los ordenes de las derivadas, expresados por el multiíndice $\alpha$ y por $m$; lo mismo que exponente $s>0$,

$$
\left|\partial_{x}^{\alpha} \mathbf{u}_{0}(x)\right| \leq \frac{C_{\alpha s}}{(1+|x|)^{s}}, \quad\left|\partial_{x}^{\alpha} \partial_{t}^{m} \mathbf{f}(x, t)\right| \leq \frac{C_{\alpha m s}}{(1+|x|+t)^{s}},
$$

Para cualquier instante $t \geq 0$, y $C$ una constante, la solución es físicamente admisible si satisface la condición de energía acotada y la regularidad de todo orden, tanto para la presión como para la velocidad del flujo:

$$
\int_{\mathbb{R}^{n}}|\mathbf{u}(x, t)|^{2} d x<C, \quad p, \mathbf{u} \in C^{\infty}\left(\mathbb{R}^{n},[0, \infty)\right), \quad t \geq 0 .
$$

El problema mencionado se plantea de la forma siguiente: para la fuerza $\mathbf{f}=0$ y la velocidad inicial $\mathbf{u}_{0}$ que satisfaga (2), demostrar la existencia de una pareja (u, $p$ ), de la velocidad de flujo y presión, suficientemente regulares, que satisfagan la ecuación de Navier-Stokes y las condiciones expresadas en (1), (3); [7].

Las aproximaciones a las soluciones de la ecuación de Navier-Stokes se obtienen por el método de las diferencias finitas o del elemento finito para discretizar las variables espaciales. Se trata de esquemas que produzcan en su forma variacional, una forma bilineal continua y coerciva, que permita aplicar el teorema de la proyección para garantizar la existencia y unicidad de la solución en su forma variacional. También existen métodos del paso fraccional y el de la compresibilidad artificial. Para la parte temporal se conocen diversos esquemas, como el que trata lo lineal en forma implícita en tanto lo no-lineal de manera explícita, o bien el Crack-Nikolson, etc., [24].

La ecuación de Navier-Stokes se reformula como la ecuación de evolución de los vórtices, que describe el movimiento local de un fluido como una composición de: una traslación rígida, una rotación rígida y una deformación; siendo el vector de rotación, la vorticidad. La ecuación de evolución para los vórtices es:

$$
\frac{\partial}{\partial t} \xi+(\mathbf{u} \cdot \nabla) \xi=\frac{1}{R} \Delta \xi+(\xi \cdot \nabla) \mathbf{u}, \quad \xi=\text { curl } \mathbf{u}, \quad \operatorname{div} \mathbf{u}=0
$$

como se observa en (4), este flujo es difusivo, así que también transporta momentum como la viscosidad; sin embargo, tiene la propiedad de ser más intenso que la difusión molecular. El régimen de escala es otra de las características más destacadas para este tipo de flujo, como lo es también, su carácter disipativo.

La ecuación de Navier-Stokes sujeta a una condición inicial puede describirse también a partir de la evolución de un vector tangente, dada por la acción de un operador de evolución ligado al flujo $\mathbf{u}$, sobre el mismo vector tangente; la parte hermítica del operador se denota por $H$ y se expresa en la ecuación (5):

$$
\begin{array}{ll}
H=\nu \Delta-w(x), & w^{2}(x)=\frac{D_{T}-1}{2 \nu D_{T}} \varepsilon(x), \\
\varepsilon(x)=\frac{\nu}{2} \sum_{i j}\left(\frac{\partial}{\partial x_{j}} u_{i}+\frac{\partial}{\partial x_{i}} u_{j}\right)^{2} &
\end{array}
$$


en donde $\nu$ es la viscosidad cinemática; $\Delta$ es el operador de Laplace; $w^{2}$ es proporcional a la tasa de disipación de la energía, por unidad de masa del flujo u, y que es denotada por $\varepsilon ;$ y $D_{T}$ es la dimensión topológica del espacio. En especial, interesa conocer la evolución en el futuro remoto, de la distancia entre dos condiciones iniciales que comienzan siendo cercanas. La estimación de la velocidad de este crecimiento se conoce como exponente característico; [14].

\section{El proceso fractal}

La turbulencia la describimos a través de una multiestructura. Para la estructura multinivel, se trata de definir, apropiadamente, una medida micro, reiterarla y conectarla con la medida macro. Lo que conduce a tres relaciones de potencia: una, entre la función de partición y la base de la resolución, potencia conocida como función de estructura; otra, entre los rasgos y la resolución, siendo la potencia, el espectro multifractal, similar a la relación entre rasgos y resolución en un fractal autosimilar; y una tercera, entre la micromedida y la base de la resolución, en donde la potencia es la dimensión local o el orden de la singularidad. Pero además, la función de estructura se convierte, con la transformada de Legendre, en el espectro multifractal. En especial, para los procesos potenciados de Bernoulli, y el modelo paramagnético, veremos que la función de partición determina la estructura.

Por una parte, para la medida multifractal en el nivel microscópico, consideramos la medida $\mu$, soportada por un conjunto autosimilar. Se conforma una malla con una colección de cubos $\left(C_{k}\right)_{k}$ de lado $l_{0} h$, que intersectan el soporte de la medida $\mu$; a $h$, la denominamos la resolución, con $0<h<1$, y a $l_{0}$, en ocasiones, la podemos tomar de valor unitario. Para una función de la posición, como lo es la velocidad, se define la medida por la norma o magnitud de sus variaciones en un desplazamiento arbitrariamente pequeño $l_{0} h$.

Se entiende como los rasgos, al número de cubos en donde la medida micro sea del orden de $\alpha$, cuando $h$ sea pequeña o como los $\alpha$-iso-cubos, [6].

La ley de escalamiento queda definida por la particularidad de que los rasgos $N_{h}(\alpha)$ son del orden de $f(\alpha)$, y por tanto, obedecen una ley de potencia, cuando la resolución se aproxima a cero.

Por el proceso de escalamiento, se pasa del nivel micro, al meso, y luego al macroscópico. Se define la función de partición por los momentos de orden arbitrario $s$. Se obtiene, entonces, una relación de potencia para la función de partición, la cual se escala como la función de estructura.

Las dos leyes de escala están relacionadas, porque la función de estructura, $\tau(s)$, es la transformada de Legendre del espectro multifractal, $f(\alpha)$; y se tiene:

$$
\tau(s)=\sup _{\bar{\alpha} \geq 0}\{f(\bar{\alpha})-s \bar{\alpha}\}, \quad \tau(s)=f(\alpha(s))-s \alpha(s) .
$$

En los fractales autosimilares, el número de rasgos crece como una potencia de la resolución. Se aplica el proceso de Cantor generalizado, se discretiza, y se obtiene la medida, que depende del índice de dimensiones $\beta$, con $\mu_{\beta}(r)=\frac{1}{\Gamma(\beta)} r^{\beta-1}$, y $h_{n}=r / n, N_{h}(\beta)=h^{-\beta}$, 
$0<\beta<1, \beta=D_{f} / D_{T}$. O visto desde lo general, un fractal autosimilar tiene un espectro que se reduce a un punto: el punto fijo, o la dimensión de información, o el orden de la sinngularidad; y que es, además, el cero absoluto del modelo termodinámico.

Para la segunda parte de la multiestructura, la turbulencia se describe por un árbol o proceso de cascada energética en el espacio tridimensional. Como árbol fractal energético, sus tallos más grandes representen los vórtices de mayor tamaño, que debido a sus interacciones mutuas, se subdividen en ramas cada vez más pequeñas; cuyo número representa la magnitud aleatoria de sus descendientes; y cuyas longitudes representan la magnitud aleatoria de sus tiempos de existencia, de tal suerte que se describan procesos de caminos aleatorios ramificados, y los cambios de escala se obtienen de la ecuación de Navier-Stokes, [19], [15], [16].

En ésta, la característica dinámica de los cambios de escala se determina por la invarianza del número de Reynolds. Este número aporta el índice de importancia entre la unidad de fuerza inercial y la unidad de fuerza viscosa: $R_{e}=v l_{0} / \nu$. La micromedida la definimos por las variaciones de la velocidad, la cual nos dice que cuando la posición cambia aritméticamente en la cantidad $l_{0} h$, la velocidad lo hace en forma geométrica, con la potencia $\alpha$, el orden de la singularidad o dimensión local,

$$
\Delta_{h l_{0}} v=v_{0} h^{\alpha}, 0<h<1,0<\alpha \leq 1 / 3 .
$$

Cuando la resolución se cambia por cierto factor, se garantiza la invarianza del número de Reynolds bajo los cambios de escala, si la viscosidad se escala por $1+\alpha$. En tanto, para la disipación de la energía se escala por $3 \alpha-1$. Se concluye que la viscosidad establece el patrón de los cambios de escala, [2]. La velocidad $v_{0}$ se tomará del modelo de Kolmogorov: $v_{0}=\left(\varepsilon l_{0}\right)^{1 / 3}$, en donde $\varepsilon$ representa la tasa de transferencia de la energía cinética y $l_{0}$ es la longitud de mezcla de Prandtl.

Para la tercera parte de la multiestructura, la ecuación de Fokker-Planck, consideramos el espacio muestral de los eventos elementales junto con la malla de cubos, que nos sirven de base para generar la sigma álgebra de sus conjuntos medibles que intersecten el soporte de la micro medida. La medida en este espacio muestral es la de Gibbs, $\nu_{k(\alpha)}(s)$. El espacio fásico será el espacio euclidiano con sus borelianos. La variable aleatoria de las variaciones de la velocidad es una función entre el espacio muestral y el fásico, con preimágenes medibles a través de la medida de Gibbs. Así que, ahora, el espacio fásico se hace también un espacio de probabilidad. Definimos el proceso estocástico indexando la variable aleatoria de las variaciones de la velocidad. La probabilidad de paso se define por la medida de Gibbs condicionada, a que el proceso se inicie en cierto punto espaciotemporal y llegue a cierto boreliano-temporal. El proceso es homogéneo con respecto al instante considerado como inicial, por lo que éste se toma como cero, y entonces, la probabilidad de paso depende del lapso de tiempo que emplea el proceso en llegar al boreliano alrededor de $x$; dependiendo también de la posición inicial y la colección de cubos. La probabilidad de paso respecto del boreliano es una medida por lo que puede hacerse integración con respecto a esta probabilidad de paso y se debe cumplir la condición de 
Chapman-Kolmogorov fraccional,

$$
\rho\left(t, x \mid x_{0}, t_{0}\right)=\int_{X} \rho(t, x \mid \bar{x}, \bar{t}) \rho\left(\bar{t}, \bar{x} \mid x_{0}, t_{0}\right) d \mu_{\beta}(\bar{x}) .
$$

Pero además, los coeficientes de memoria de este proceso se definen por

$$
D^{(n)}(x, t)=\lim _{\Delta t \rightarrow 0} \int_{X} \rho(\bar{x}, t+\Delta t \mid x, t) d \mu_{\beta}(\bar{x}) \frac{1}{n !} \frac{\left(\bar{x}^{\beta}-x^{\beta}\right)^{n}}{\Delta t} .
$$

En particular, interesan los coeficientes de memoria de orden 1, llamado coeficiente de arrastre; el de orden 2, llamado coeficiente de difusión; y los de orden 3 o superior, los cuales son nulos, por la conocida expansión de Kramers-Moyal; con lo que se tiene otra condición para la probabilidad de paso, porque sus coeficientes de memoria, para los ordenes de tres y superiores, deben tender a cero, para tiempos de transición muy cortos, [23], [17].

La ley de evolución, ecuación de Fokker-Planck, y el operador de la ley de flujo dependiente de los coeficientes de difusión y arrastre, y del índice de dimensiones $\beta$, se muestran a la izquierda de la ecuación (9); y las soluciones estacionarias, debajo de la misma,

$$
\begin{aligned}
& \frac{\partial}{\partial t} \rho(x, t)=\frac{\partial}{\partial x^{\beta}}[q(\rho(x, t))], \quad q=\frac{\partial}{\partial x^{\beta}}(D(x, t)(\cdot))-a(x, t)(\cdot), \\
& \rho(x, t)=\frac{c t e}{D(x, t)} e^{\int \frac{a}{D} d x^{\beta}} .
\end{aligned}
$$

\section{Modelos de turbulencia}

\subsection{Modelo de extremos}

Nuestro primer enfoque consiste en suponer que la distribución de la variable aleatoria se concibe como límite de una sucesión de variables aleatorias estacionarias, e imaginar que, en sus interacciones mutuas, los intentos de transferencia entre unos vórtices y otros, no pueden fructificar hasta que se alcance un número suficiente de ellos; si se disminuye la escala del tiempo, la probabilidad del éxito de la transferencia crece exponencialmente en tanto la del fracaso, lo hace como potencia; con una ulterior disminución de la escala del tiempo, la probabilidad de éxito crece como una exponencial reforzada o potenciada, entonces se distribuye como los extremos, o según una variable aleatoria Weibull-Gnedenko, en tanto que el fracaso se comporta como una función de riesgo. De tal suerte, que introducimos una asimetría entre las tendencias del éxito y el fracaso en la transferencia de energía. Para lo que fijamos los coeficientes de memoria como se muestran en la ecuación (10); y resulta, entonces, la solución estacionaria, la que se muestra debajo de (10); [23], [9]; por lo que la solución estacionaria resulta,

$$
\begin{array}{ll}
a(x)=a_{1}|x|^{1-\alpha_{F}}, & D(x)=D_{1}|x|^{1-\gamma}, \\
\rho=\operatorname{cte}(x)^{-(1-\gamma)} \exp \left(\frac{\alpha_{F} a_{1}}{(1+\gamma) D_{1}}|x|^{\gamma}\right) . &
\end{array}
$$

Escogiendo apropiadamente las constantes de la solución estacionaria, y reiterando el planteamiento de que la primera variable aleatoria se distribuya como los extremos, con 
$\lambda=3$ como parámetro de escala $\mathrm{y}, \gamma>0$ como el parámetro de forma; y la otra variable aleatoria se distribuya como una función potencia: $\gamma_{2} s^{\gamma_{2}-1}, \gamma_{2}>0$, con el mismo parámetro de escala. Entonces, la conjunción de las dos variables se distribuye como el producto; por lo que el número de rasgos es como en la ecuación (11):

$$
N=\gamma_{2} s^{-\left(1-\gamma_{2}\right)} \cdot \gamma s^{-(1-\gamma)} e^{s^{\gamma}}, s=\bar{s} / 3
$$

Se obtienen el espectro de dimensiones, las dimensiones locales u órdenes de las singularidades y la función de estructura; que se muestran en la ecuación (12). En donde la condición de Kolmogorov puede verse como: $\bar{s}=3, \tau(3 / 3)=\tau(1)=0$, la cual coincide con la condición de normalización para la función de estructura. Para el parámetro de forma $\gamma$, con $\gamma+\gamma_{2}=2$, resulta, por tanto, la ecuación a la derecha de (12),

$$
\begin{array}{ll}
\alpha(s)=D_{B}+\frac{C}{\gamma-1}\left(1-\gamma s^{\gamma-1}\right), & f(\alpha(s))=D_{B}-C s^{\gamma} \\
\tau(s)=D_{B}(1-s)+\frac{C}{\gamma-1}\left(s^{\gamma}-s\right), \gamma \neq 1, & \gamma=1+\sqrt{1-h^{D_{B}}}
\end{array}
$$

Así que fijada la resolución y la dimensión caja del soporte, se obtiene el parámetro de forma; el cual debe ser, entonces, mayor que 1 . Fijado $h, \gamma$ crece si y solo si lo hace $D_{B}$. Puede comprobarse que cuando $\gamma$ se acerca a 2, la densidad de la distribución se acerca a una log-gaussiana, cuya varianza decrece con el crecimiento de la dimensión $D_{B}$.

El parámetro que nos interesa es el índice de dimensiones u ocupación del espacio, por lo que los rasgos lo renormalizamos considerando que cuando $s=0$, el número de rasgos debe ser, como en el conjunto de Cantor, la unidad,

$$
N_{h}(\alpha(s))=h^{\left(\frac{s}{3}\right)^{\gamma}}
$$

en particular, se observa que cuando se acerca a 2 y la resolución a $e^{-1}$ entonces el número de rasgos se distribuye como una gaussiana de media cero y varianza $9 / 2$. Por tanto, vemos que esta distribución engloba a la gaussiana; pero, una generalización de ésta última es el concepto de distribución Lévy.

En tanto movimientos, el punto de partida puede considerarse en el movimiento browniano de incrementos independientes que se distribuyen como una gaussiana estándar; concepto que puede extenderse, al menos, de dos formas; una, manteniendo la distribución gaussiana de los incrementos pero suprimiendo su independencia, con lo que obtenemos los movimientos brownianos fraccionarios (fBm); dos, manteniendo la independencia de los incrementos, pero que se distribuyan como una Lévy estable (sLm); también conocido por (fLm), movimiento Lévy fraccionario. Un movimiento Lévy estable simétrico, con parámetro de localización nulo, e índice de estabilidad $\gamma, 0<\gamma \leq 2$, tiene una función característica como la ecuación (13), para el número de rasgos.

La ecuación (13) contiene un doble carácter; por una parte, es la función característica, en la variable de Fourier $s$, de una distribución Lévy; por otra, es la distribución extrema de Weibull-Gnedenko.

Pero además, como movimiento sLm, es un proceso autoafín, con índice de autoafinidad igual a $1 / \alpha$; y tiene media infinita en el intervalo $0<\gamma \leq 1$, media finita $1<\gamma \leq 2$, varianza infinita $0<\gamma<2$, y sólo varianza finita para $\gamma=2$. 
La distribución Lévy no tiene una expresión en términos de funciones analíticas usuales, salvo para índices particulares, pero sí cuenta con una representación en su expansión como serie

$$
\frac{1}{\Gamma\left(\frac{1}{\gamma}\right)} \sum_{j=0}^{\infty}(-1)^{j} \frac{\Gamma\left(\frac{j+1}{\gamma}\right)}{j !}\left(\frac{s}{3}\right)^{j} \cos \frac{1}{2} j \pi
$$

Comparamos la distribución multifractal con una distribución Lévy, normalizada por $F(0)=1$; buscando una Lévy que la aproxime. Encontramos que así lo hace una Lévy con índice de estabilidad igual al parámetro de forma (1.92); por lo que obtenemos una nueva interpretación y nombre para el parámetro de forma: es el índice de estabilidad Lévy. Además, la cercanía de las dos distribuciones nos permite obtener la función característica, aproximada, para la distribución multifractal: $f_{c}(k) \approx e^{-|k|^{\gamma}}$.

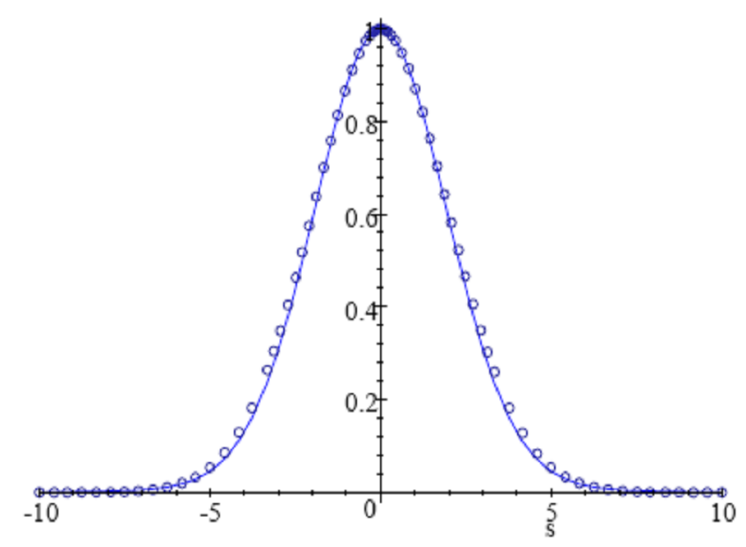

Figura 1: Densidades Multifractal y Lévy.

En la figura 1, mostramos la multifractal en café con bolitas; y la Lévy, en línea roja continua. En dicha gráfica, una franja vertical de ancho $d s$, alrededor de $s$, representa al índice de rasgos o el número de cubos en donde la medida es del orden de $\alpha(s)$. Por ejemplo, según la multifractal, aproximadamente el $87.5 \%$ de los cubos tienen medida del orden de la dimensión de información $\alpha(1)=0.7052$, con dimensión del soporte de 1.7052, y resolución $1 / 3$.

Resulta, también, particularmente interesante calcular la dimensión generalizada y el índice de intermitencia para este modelo de turbulencia. El resultado se muestra en la ecuación (15). En donde, observamos que cuando el índice decrece hacia 1, la intermitencia se incrementa sin límite, en tanto cuando éste crece hacia 2, la intermitencia decrece a su mínimo valor posible: $C$. Con lo que obtenemos, además, una interpretación física para la constante $C$, y una reinterpretación para la dimensión de información como la diferencia entre la máxima dimensión fractal y la mínima intermitencia, la cual se obtiene para la 
gaussiana, [8],

$$
D_{s}=\frac{\tau(s)}{1-s}=D_{B}+\frac{C}{\gamma-1}\left(\frac{s^{\gamma}-s}{1-s}\right), \iota=-\left[\frac{\partial}{\partial s} D_{s}\right]_{s=0}=\frac{C}{\gamma-1}
$$

\section{Parte II: Otros modelos}

\subsection{Procesos potenciados de Bernoulli}

La turbulencia también la podemos imaginar como un sistema de distribución de energía cinética, y describirlo como una colección de vórtices interactúantes con un aspecto esparcido e irregular; cubierto con la malla de cubos de un tamaño homogéneo, pero variable; $\mathrm{y}$ al que queremos determinar su espectro de dimensiones fractales.

Como cuerpo macroscópico seguimos el método termodinámico y lo estudiamos como procesos potenciados de Bernoulli. Al cuerpo lo consideramos como compuesto de varios estados microscópicos. Definimos el número $p_{i}$, como probabilidad de éxito en la transferencia de energía, siendo $\sum p_{i}=1$, y $0<p_{i}<1$. Definimos una medida microscópica por $\mu_{i}=p_{i}$. El paso de un nivel micro a uno macro lo damos a través de la reiteración, por lo que potenciamos las probabilidades de transmisión, definiendo: $\nu_{i}(s)$, los cuales se complementan, en el sentido de que satisfagan: $\sum \nu_{i}(s)=1,0<\nu_{i}(s)<1$; y que se recuperan las medidas micro por $\nu_{i}(1)=p_{i}$. Estos se definen en la ecuación (16).

Vamos a suponer que tratamos con un cuerpo que cuenta con $K$ estados microscópicos. Después de $s$ reiteraciones del proceso inverso a la pulverización, el cuerpo se encontrará en un estado macroscópico, cuya entropía, según definición, viene dada por:

$$
f(\alpha)=\sum_{k} \nu_{k} \log _{h} \nu_{k}, \nu_{k}(s)=\frac{p_{k}^{s}}{\sum_{i} p_{i}^{s}} .
$$

Aplicando las propiedades del logaritmo descomponemos la entropía en sus partes, lineal con $s$ y su expresión complementaria, con lo que obtenemos las expresiones para la distribución de singularidades y para la función de estructura,

$$
\begin{gathered}
f(\alpha)=\sum_{k} \log _{h}\left(\nu_{k}\right)^{\nu_{k}}=\log _{h} \prod_{k}\left(p_{i}^{s} /\left(\sum_{i} p_{i}^{s}\right)\right)^{\nu_{k}} \\
=-\log _{h} \sum_{i} p_{i}^{s}+s \sum_{k} \nu_{k} \log _{h} p_{k} ; \\
\alpha(s)=\sum_{k} \nu_{k} \log _{h} p_{k} ; \tau(s)=-\log _{h} \sum_{i} p_{i}^{s} .
\end{gathered}
$$

Pero en términos de la función de partición o suma estadística se tiene, para la función de estructura; la distribución de singularidades; y la entropía, las expresiones dadas en (19),

$$
\tau(s)=-\log _{h} \frac{Z(s)}{(Z(1))^{s}} ; \alpha(s)=-\partial_{s}(\tau(s)) ; f(\alpha)=\tau(s)+s \alpha(s) .
$$


Esta expresión engloba la relación clásica para la entropía en la termodinámica, ya que la misma se reproduce cuando la resolución se toma como $h=e^{-1}$, y la potencia de reiteración se la hace como $s=1 / T$, siendo $T$ la temperatura.

Por otra parte, si partimos de estas relaciones (19) y asumimos que la función de partición se determina de dos micro-estados, medidos por la probabilidad de éxito y la de fracaso, se tiene para la misma: $Z(s)=p^{s}+q^{s}, p+q=1$, y reobtenemos las expresiones dadas en (17 y 18); pero, para un sistema compuesto sólo de dos micro-estados.

En la figura 2, representamos el orden de las singularidades $\alpha$, en forma paramétrica y $l^{\prime}$ inea cafde forma sigmoide, $(\alpha(s, 1 / 7,3), s)$; la inversa del espectro multifractal con línea roja y forma acampanada, $(f(s, 1 / 7,3), s)$; y la dimensión de información con línea negra y círculo. La resolución es $1 / 3$, y la probabilidad de éxito $1 / 7$.

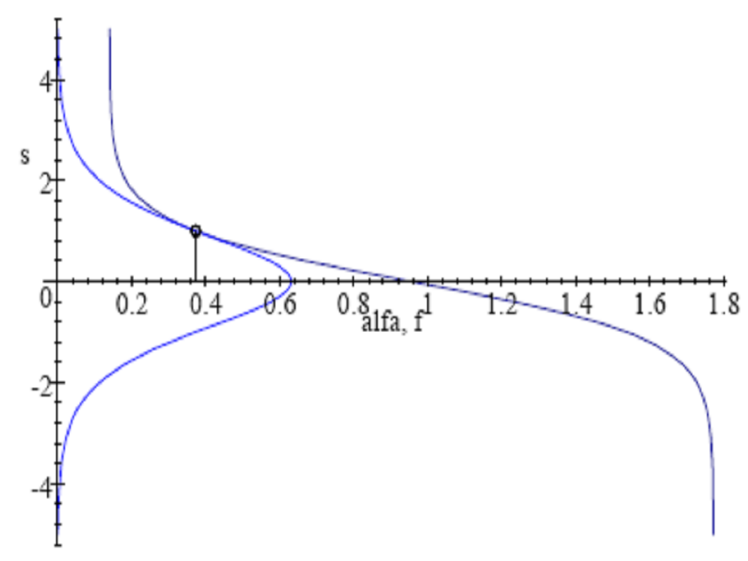

Figura 2: Multifractal y singularidades.

\subsection{Modelo paramagnético}

La interacción entre dos vórtices puede describirse por una ley de Biot-Savart, en la cual la interacción varía con el cuadrado de la distancia; así que vórtices más cercanos tienen una interacción más intensa. Cuando los vórtices son más grandes la interacción es menor, incluso entre vórtices más cercanos, pero a medida que los vórtices son más pequeños crece la interacción, la energía es mayor en valor absoluto; por tanto, la probabilidad de la fractura del vórtice se incrementa y su parámetro de forma tiene que ser mayor que 1.

Es ampliamente conocida la ley experimental que encontró Pierre Curie para la magnetización de un material paramagnético en el caso extremo de altas temperaturas, en 1895; y que luego, su alumno Paul Langevin extendió para temperaturas moderadas. Se trata de un sistema con dos estados micros, uno paralelo al campo externo, con energía proporcional a $-\lambda$; y el otro, antiparalelo, con energía proporcional a $\lambda$. Fijamos la resolución en $h=e^{-1}$, y la función de partición resulta: $Z(s)=e^{-\lambda s}+e^{\lambda s}=2 \cosh \lambda s$, luego 
la función de estructura, la distribución de singularidades y el espectro son:

$$
\begin{aligned}
\tau(s) & =\ln \left(\frac{2 \cosh \lambda s}{(2 \cosh \lambda)^{s}}\right) ; \alpha(s)=-\lambda \tanh \lambda s+\ln (2 \cosh \lambda) \\
f(\alpha(s)) & =\ln \left(\frac{2 \cosh \lambda s}{(2 \cosh \lambda)^{s}}\right)+s(-\lambda \tanh \lambda s+\ln (2 \cosh \lambda)) .
\end{aligned}
$$

\section{4 Árbol o cascada energética}

Describimos el árbol o cascada energética, para hallar el espectro de energía transferida y su relación con el espectro multifractal.

Queremos recordar que el llamado subrango inercial es aquel en donde la producción y disipación de la energía cinética turbulenta es casi nula. Este rango puede ser muy amplio cuando el número de Reynolds es lo suficientemente alto, y termina cuando la etapa de disipación empieza a ser significante. Éste es precisamente el caso de la llamada turbulencia completamente desarrollada.

La cascada se inicia con cierto tamaño y número de los vórtices o edis, $\left(l_{0}, N_{0}\right)$, los cuales contienen cierta energía. Esos edis, a través de la interacción mutua, se fraccionan en otros cada vez más pequeños, pero también cada vez más numerosos y la energía se transfiere de un nivel al siguiente. Se asume que el tamaño de los edis está representado a través de la resolución, en tanto el número de los edis, por los rasgos, $N_{h}(\alpha)$. En el proceso, se hace cada vez más pequeña la resolución, en tanto, simultáneamente, se incrementa el número de los rasgos y la energía se distribuye entre un número mayor de ellos.

Se empieza por estimar la energía transferida acumulada en la etapa $n$ del proceso de fractalización. Si $\varepsilon$ denota la tasa de transferencia de la energía de una escala a la siguiente en el proceso de fractalización. Ésta se estima por el cociente entre la cantidad de energía al nivel $n$, y el tiempo de existencia de ese nivel, $\Delta t_{n}$; el cual a su vez, se aproxima por el cociente entre tamaño de los vórtices y la fluctuación cuadrática media de la velocidad; con $h_{n}=l_{0} h^{n}$, se tiene:

$$
\begin{gathered}
\varepsilon=\frac{I_{n}}{\Delta t_{n}}=\frac{1}{\Delta t_{n}} \int_{k_{n}}^{k_{n+1}} E(k) d k, \quad \frac{h_{n}}{\Delta t_{n}}=\left\langle\left|u\left(x+h_{n}\right)-u(x)\right|^{2}\right\rangle^{\frac{1}{2}}, \\
N_{h}(\alpha)=h^{-f(\alpha)}, \quad I_{n}=\left(\frac{N_{h}(\alpha)}{h^{-D} T}\right)^{n}\left\langle\left|u\left(x+h_{n}\right)-u(x)\right|^{2}\right\rangle, \\
\varepsilon=\frac{1}{l_{0}} h^{-n\left(1+\frac{D_{T}-f(a)}{2}\right)}\left(\int_{k_{n}}^{k_{n+1}} E(k) d k\right)^{\frac{1}{2}+1}, \\
\int_{k_{n}}^{k_{n+1}} E(k) d k=\left(\varepsilon l_{0}\right)^{\frac{2}{3}}\left(h^{n}\right)^{\frac{2}{3}+\frac{D_{T}}{3}(1-\beta(\alpha))} ;
\end{gathered}
$$

por tanto,

$$
E(k)=C_{1} \varepsilon^{\frac{2}{3}}\left(l_{0}\right)^{-\frac{C s}{3}}(k)^{-\frac{5}{3}-\frac{C s}{3}} .
$$

Se obtiene, entonces, el espectro de la energía transferida, el cual se ve como una generalización del histórico resultado de Kolmogorov. El mismo se muestra en la ecuación (24) 
para el caso unifractal, en donde el espectro se colapsa en un solo punto: $f(\alpha)=\alpha=D_{f}$. Se observa que se cumple la condición de Kolmogorov cuando el índice de dimensiones asume el valor 1, y desaparece la intermitencia,

$$
d E_{\eta}=\left(\frac{8}{3}-\beta\right) k^{-\frac{5}{3}}(k)^{-(1-\beta)} ; \beta=\frac{D_{f}}{D_{T}} .
$$

En el proceso anterior identificamos un parámetro de forma que llamamos $a=\frac{2}{3}+$ $\frac{D_{T}}{3}(1-\beta)+1=\frac{8}{3}-\beta, D_{T}=3$; sin embargo, se puede argumentar que ese análisis es unilateral, porque no se ha tomado en cuenta la posibilidad del fracaso en el traspaso de energía; para ello, entonces, debemos considerar otro parámetro que simbolizamos como $b$ y que vincularemos con cierta curvatura más adelante. Así las cosas, contamos con dos parámetros positivos: $a>0, b>0$. Recordando la razón de oro, que puede enunciarse diciendo que el mayor de los dos parámetros es media proporcional entre la totalidad o suma, y su complemento, $\frac{b+a}{a}=\frac{a}{b}$, suponiendo que $a>b$, aunque no es una restricción necesaria; pero que también podemos enunciar diciendo que la probabilidad a favor de un evento es igual a su pronóstico en contra $\frac{a}{b+a}=\frac{b}{a}$. Definimos una sucesión, basados en estos parámetros, por: $\frac{(b+a)_{j}}{(a)_{j}}$, en donde $(a)_{j}=a(a+1)(a+2) \ldots(a+j-1)=\frac{(a+j-1) !}{(a-1) !}=$ $\frac{\Gamma(a+j)}{\Gamma(a)}$ son los símbolos de Pochhammmer. Sea la sucesión de los rasgos proporcional a la potencia siguiente de la resolución, en donde la proporcionalidad se da por la sucesión de las razones de oro de los dos parámetros,

$$
p_{j}=\frac{(a+b)_{j}}{(a)_{j}} h^{-(j+1)} \text {. }
$$

A través de un proceso de Cantor generalizado, el cual se realiza por medio del algoritmo que se muestra a la izquierda de (26), cuando se aplica a la expresión en (25); para luego discretizar, escogiendo la sucesión de resoluciones como: $h_{n}=\frac{n}{k^{2}}$; y finalmente, llevarla al límite; con lo que se obtiene la expresión debajo de (26),

$$
\begin{aligned}
P_{n, j} & =\left((-1)^{n} \frac{d^{n}}{d h^{n}}\left(\frac{(a)_{j}}{(a+b)_{j}} h^{(j+1)}\right) \cdot(n+1) \partial_{p_{j}}^{-n}\left(p_{j}\right)\right) \\
P_{j} & =\frac{(a)_{j}}{(a+b)_{j}} \frac{1}{\Gamma(j+1)}\left(k^{2}\right)^{j}
\end{aligned}
$$

luego, con $a+b \neq 0,-1, \ldots,-j$

$$
\sum_{0}^{\infty} P_{j}=\sum_{0}^{\infty} \frac{(a)_{j}}{(a+b)_{j}} \frac{\left(k^{2}\right)^{j}}{\Gamma(j+1)}=M\left(k^{2} ; a, a+b\right),
$$

en donde la serie es convergente para todo $k$ finito. Pero ésta es la función confluente de Kummer $M(i k ; a, a+b), \operatorname{Re}(a)>0, \operatorname{Re}(b)>0$; para la cual, contamos con la repre- 
sentación integral:

$$
\begin{aligned}
M(i k ; a, a+b) & =\int_{0}^{1} \frac{h^{a-1}(1-h)^{b-1}}{B(a, b)} e^{i k h} d h \\
& =\int_{0}^{1} B \operatorname{den}\left(h ; \frac{8}{3}-\beta, b\right) e^{i k h} d h ;
\end{aligned}
$$

en donde Bden es la densidad de la distribución Beta. Por tanto, la función de correlación resulta ser la trasformada de Laplace de la densidad Beta:

$$
U(r)=M\left((i r)^{2} ; a, a+b\right)=\int_{0}^{1} B d e n\left(h ; \frac{8}{3}-\beta, b\right) e^{-r^{2} h} d h .
$$

Para que la distribución Beta tenga la forma unimodal se requiere que sus dos parámetros sean mayores que 1 . El primero, $a=\frac{8}{3}-\beta$, evidentemente lo es, porque su mínimo valor posible se obtiene para el máximo valor posible del índice de dimensiones el cual es $1, y$, entonces, para este valor se tiene $a=\frac{5}{3}>1$. Para el segundo, buscaremos las condiciones que garantizan esta restricción. Para ello analizamos la expresión para la función de correlación. La normalización de la Beta se traduce en $U(0)=1$. La pendiente en el origen produce $U^{\prime}(0)=0$; en tanto que la curvatura en el origen es proporcional al primer momento de la Beta y la simbolizamos por $\kappa, U^{\prime \prime}(0)=-2 \frac{a}{a+b}=-\kappa$, luego el segundo parámetro depende de esta curvatura, y se expresa por $b=a\left(\frac{2}{\kappa}-1\right)$. Aproximamos la transformada de Laplace de la función de correlación, por una distribución Lévy, y definimos la curvatura por el valor que asume la misma:

$$
\left[-\frac{\partial^{2}}{\partial r^{2}} \frac{1}{\Gamma\left(\frac{1}{\gamma}\right)} \sum_{j=0}^{\infty}(-1)^{j} \frac{\Gamma\left(\frac{j+1}{\gamma}\right)}{j !} r^{j} \cos \frac{1}{2} j \pi\right]_{r=0}=\frac{\Gamma\left(\frac{3}{\gamma}\right)}{\Gamma\left(\frac{1}{\gamma}\right)}=\kappa ;
$$

entonces el segundo parámetro de forma para la densidad Beta será como en (29); se determina por el índice de estabilidad Lévy, pero como también el índice de dimensiones depende de este índice, la forma de la densidad Beta vendrá determinada por el índice de estabilidad.

Cuando la curvatura es menor que 1, el primer parámetro de forma es menor que el segundo, $a<b$, y como el primero es mayor que 1, luego también lo es el segundo. Por otra parte, cuando la curvatura recorre el rango: $1 \leq \kappa<2$, entonces el índice Lévy lo hace en el rango: $1.286 \geq \gamma>1$. Para un valor del índice de dimensiones $\beta$, en un gráfico de $b(\beta, \gamma)$ puede verse que $b$ se incrementa cuando $\beta$ disminuye, de tal forma que entre menor sea la ocupación del espacio, mayores serán los valores que pueda asumir el parámetro $b$; por lo que éste resulta mayor que la unidad, dando lugar a la forma unimodal de la densidad Beta.

En particular, cuando $\gamma$ es cercano a la unidad, como es el caso de la proporción de oro cuando es la media proporcional entre la totalidad y la menor, debe tenerse: $\frac{a+b}{a}=$ 


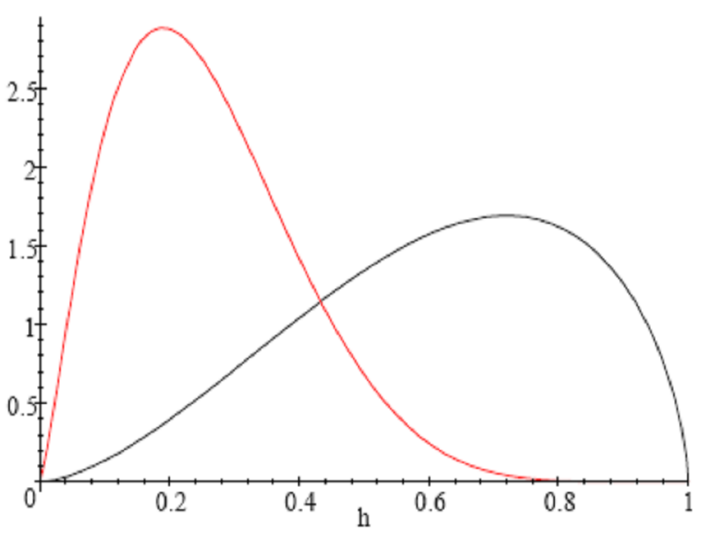

Figura 3: Densidades Beta.

$\frac{a}{b}=\frac{2}{\kappa}=2 \frac{\Gamma\left(\frac{1}{\gamma}\right)}{\Gamma\left(\frac{3}{\gamma}\right)}=\frac{1+\sqrt{5}}{2}$, entonces el índice Lévy debe ser: $\gamma=1.1773 ;$ y para una ocupación del espacio, que sea suficientemente pequeña, se tiene: $a=\frac{8}{3}-\frac{1}{1000}=2.7$, y $b=1.647$; lo que da lugar a la densidad Beta: $\frac{h^{1.7}(1-h)^{0.647}}{B(2.7,1.647)}$. Por el contrario cerca del otro extremo, cuando $\gamma$ es cercano a 2, por ejemplo $\gamma=1.9931$, la distribución es $\frac{h^{\frac{4}{3}}(1-h)^{\frac{17}{3}}}{B\left(\frac{7}{3}, \frac{20}{3}\right)}$. Las densidades mencionadas se muestran en la figura 3; en donde se observa que cuando el índice Lévy se acerca a la unidad, la gráfica se desplaza hacia la derecha, aumentando el valor de la moda, en tanto cuando este índice se acerca a 2, la moda se desplaza hacia la izquierda y disminuye.

\subsection{Modelo de Pearson}

Una de las características más importantes de la turbulencia es la mezcla y coexistencia de una gran diversidad de escalas. Las escalas pequeñas presentan las dificultades mayores para su modelación. Tanto los resultados de laboratorio, como las simulaciones numéricas, muestran la naturaleza no-gaussiana de las escalas pequeñas, con distribuciones de los gradientes de velocidad que tienen como características el ser más picudas y poseer colas más gordas. Las colas más gordas se reflejan en la asimetría, la cual se mide por el tercer momento central, de suerte que las simetrías positivas, se corresponden con colas más gordas a la derecha que a la izquierda. En tanto que, el cuarto momento, o curtosis, se manifiesta en distribuciones más picudas y colas más gordas. Pero, la curtosis alta se interpreta admitiendo que la varianza se está nutriendo de desviaciones extremas, aunque poco frecuentes; mientras la curtosis baja se la interpreta diciendo que la varianza está recibiendo desviaciones muy frecuentes, pero relativamente modestas.

La distribución gaussiana tiene una generalización en las distribuciones de Pearson. La distribución gaussiana resurge a bajos números de Reynolds, y es la distribución de las variaciones de la velocidad en el modelo de turbulencia de Kolmogorov. Por tanto, 
buscamos una distribución, que a través del método de Pearson, englobe a la distribución gaussiana. Consideramos el intervalo que determina el rango inercial, con $k_{0}$ la mínima y $k_{\eta}$ la máxima, con las relaciones $\omega_{0} \propto k_{0}^{-1}$ y $\omega_{\eta} \propto k_{\eta}^{-1}$ que pueden representar la raíz cuadrada de la energía cinética de rotación a la escala de los vórtices grandes, $l_{0}$; y la de los vórtices pequeños, $\eta$, en donde se inicia la disipación.

Karl Pearson, 1895, trabajó con densidades de probabilidad determinadas por un sistema de cuatro parámetros, que resuelven la ecuación diferencial, en donde la derivada logarítmica se expresa como un polinomio racional, dado por el cociente entre uno lineal y otro cuadrático. La variable $x$ la concebimos como frecuencia angular de rotación, [1], $[10]$,

$$
\frac{d}{d x} \ln \rho=\frac{P(x)}{Q(x)}=\frac{a_{0}+a_{1} x}{b_{0}+b_{1} x+b_{2} x^{2}} .
$$

Los cuatro parámetros pueden ponerse en correspondencia con los cuatro primeros momentos centrales de la distribución por lo que se determinan mutuamente. El polinomio cuadrático se descompone por sus raíces; vistas como fluctuaciones de las frecuencias angulares de rotación, que recorren desde sus valores relativamente bajos para los vórtices grandes en donde se inicia la cascada energética, hasta sus valores relativamente altos de los vórtices más pequeños en donde termina la cascada y la energía sale disipada. Cuando éstas sean reales y distintas, el cociente se representa por sus fracciones parciales,

$$
\begin{aligned}
\frac{P(x)}{Q(x)} & =\frac{a_{0}+a_{1} x}{\left(x+\omega_{\eta}\right)\left(x-\omega_{0}\right)}=\frac{n}{x+\omega_{\eta}}+\frac{m}{x-\omega_{0}}, \\
n & =\frac{-a_{0}+a_{1} \omega_{\eta}}{\omega_{\eta}+\omega_{0}}>-1 ; m=\frac{-a_{0}+a_{1} \omega_{0}}{\omega_{\eta}+\omega_{0}}>-1 .
\end{aligned}
$$

Lo que produce, con $C$ una constante de normalización,

$$
\rho(x)=C\left(x+\omega_{\eta}\right)^{n}\left(x-\omega_{0}\right)^{m}, \frac{x+\omega_{\eta}}{\omega_{\eta}+\omega_{0}}=h, \frac{-x+\omega_{\eta}}{\omega_{\eta}+\omega_{0}}=1-h,
$$

y se reproduce la distribución Beta con la normalización apropiada y la identificación de los parámetros de forma en términos de los coeficientes que realizan la descomposición en fracciones parciales del polinomio racional; por lo que las escalas macro y la micro determinan los parámetros de forma,

$$
\rho(h ; a, b)=\frac{1}{B(a, b)} h^{a-1}(1-h)^{b-1}, a=n+1, b=m+1 .
$$

Los coeficientes del polinomio cuadrático del denominador se determinan de sus raíces, por lo que el discriminante y el parámetro se observa que son negativos, como corresponde a las distribuciones de Pearson de tipo I, y se muestran en (35), con $b_{2}=1$,

$$
\begin{array}{ll}
b_{0}=-\omega_{\eta} \omega_{0}, & b_{1}=-\frac{1}{2}\left(\omega_{0}-\omega_{\eta}\right), \\
D=b_{0} b_{2}-b_{1}^{2}=-\frac{1}{2}\left(\omega_{\eta}^{2}+\omega_{0}^{2}\right)<0, & \lambda=\frac{b_{1}^{2}}{b_{0} b_{2}}=-\frac{1}{4} \frac{\left(\omega_{0}-\omega_{\eta}\right)^{2}}{\omega_{\eta} \omega_{0}}<0 .
\end{array}
$$

Pero además, para la normalización $a_{1}=1$, los coeficientes del polinomio cuadrático se determinan de los cuatro primeros momentos centrales; o bien, debido a la condición sobre 
el segundo coeficiente, el número de grados de libertad se reduce en 1 , y dependen de la varianza, en tanto segundo momento central, $\mu_{2}$; de la asimetría y de la curtosis, $\beta_{1}$ y $\beta_{2}$; los cuales a su vez, se obtienen de los dos parámetros de forma $a$ y $b$, mismos que se determinan de los índices Lévy y de dimensiones, $\gamma$ y $\beta(\alpha)$. Por lo que finalmente, las dos raíces, singularidades de la densidad de la distribución de Pearson, solución de su ecuación diferencial, se determinan de éstos dos índices,

$$
\begin{array}{ll}
2 b_{1}=-a_{0}=-\frac{\mu_{2}^{1 / 2}}{A^{\prime}} \beta_{1}\left(\beta_{2}+3\right), & b_{0}=-\frac{\mu_{2}}{A^{\prime}}\left(4 \beta_{2}-3 \beta_{1}^{2}\right) \\
b_{2}=-\frac{1}{A^{\prime}}\left(2\left(\beta_{2}-3\right)-3 \beta_{1}^{2}\right)=1, & A^{\prime}=10 \beta_{2}-18-12 \beta_{1}^{2} .
\end{array}
$$

La distribución de Gauss se recupera de la asimetría 0 y curtosis 3, lo que se traduce en $b_{2}=0=b_{1}, b_{0}=-\mu_{2}$.

En un plano determinado por la asimetría, en la horizontal; y la curtosis, en la vertical, la condición sobre $b_{2}=1$ determina una parábola, la parábola de referencia (linea continua), figura [4]. El parámetro $A$ determina otra parábola (círculos rojos), en cuya región positiva (convexa) cae la parábola de referencia; por lo que sobre ella, $A$ es positiva. Entonces para que $b_{1}$ sea negativa, se requiere que la asimetría resulte negativa, lo que se traduce en que las colas deben ser más gruesas a la derecha que a la izquierda. Por otra parte, $b_{0}$ también debe ser negativa, por lo que se determina una parábola $\beta_{2}=\frac{3}{4} \beta_{1}^{2}$ (cruces azules), en cuya región convexa se satisface la condición para $b_{0}$, porque en ésta se encuentra la curva de referencia. La parábola $\beta_{2}=\frac{3}{4} \beta_{1}^{2}-3$ (diamantes amarillos), aporta otra región positiva por arriba de ella, en donde cae también, la curva de referencia, por lo que la curtosis sobre ésta resulta mayor que 3; lo cual se traduce en una distribución de tipo leptocúrtica de colas más gruesas.

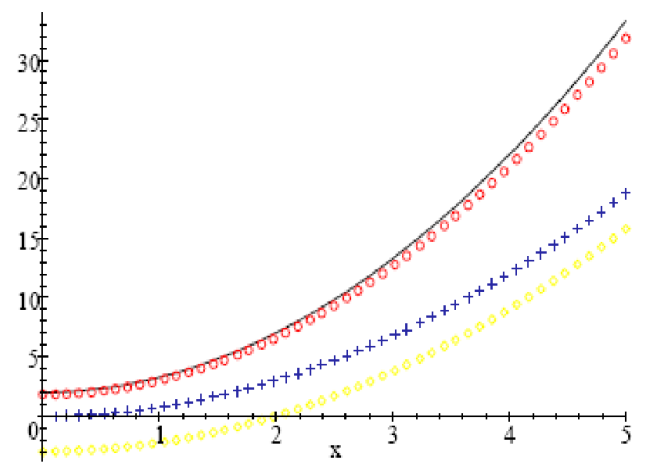

Figura 4: Curtosis vs Asimetría.

Observando que los coeficientes del polinomio cuadrático resultan ser funciones de la varianza, la asimetría y la curtosis, se puede replantear el problema con coeficientes redefinidos como en (37); en donde se hace más sencilla y se resalta la dependencia de 
estos coeficientes con los parámetros de la asimetría y la curtosis,

$$
\begin{array}{lll}
b_{1} \mapsto \bar{b}_{1}=-\frac{1}{2}\left(\omega_{0}-\omega_{\eta}\right) \frac{A^{\prime}}{\mu_{2}^{1 / 2}}, & \mapsto \bar{b}_{1}=-\frac{1}{2} \beta_{1}\left(\beta_{2}+3\right), \\
b_{0} \mapsto \bar{b}_{0}=-\omega_{\eta} \omega_{0} \frac{A^{\prime}}{\mu_{2}}, & \mapsto \bar{b}_{0}=-4 \beta_{2}+3 \beta_{1}^{2} .
\end{array}
$$

\subsection{La distribución de los gradientes}

Dado que conocemos la distribución de las variaciones o diferencias de velocidades en términos de la resolución con dos parámetros de forma, dependientes del índice de dimensiones y del radio de curvatura del espectro en el origen, podemos plantearnos representar la distribución de los gradientes de velocidad, confirmando la distribución gaussiana o normal, como la distribución asintótica para los vórtices de mayor tamaño, [2]. Representamos por $\phi$ los gradientes de velocidad, luego $l_{0} \phi$ tiene las dimensiones de la diferencia de velocidades, por lo que escribimos $\left|\partial_{x^{j}} v_{i}\right|=\phi \longleftrightarrow\left|\frac{\Delta_{h} v}{h l_{0}}\right|$, y con el cambio de variable: $v_{0}=\frac{\nu R_{e}}{l_{0}}\left(l_{0} \phi\right)^{p}$, para cierto exponente $p$, que queremos determinar; luego $d v_{0}=\frac{\nu R_{e}}{l_{0}} p\left(l_{0} \phi\right)^{p-1} d\left(l_{0} \phi\right)$. Así que:

$$
\begin{aligned}
\exp \left(-\frac{v_{0}^{2}}{2 \sigma^{2}}\right) & \mapsto \exp \left(-\frac{\left(\frac{\nu R_{e}}{l_{0}}\left(l_{0} \phi\right)^{p}\right)^{2}}{2 \sigma^{2}}\right) \frac{\partial\left(v_{0}\right)}{\partial\left(l_{0} \phi\right)} \\
& =\frac{\nu R_{e}}{l_{0}} p\left(l_{0} \phi\right)^{p-1} \exp \left(-\frac{\left(l_{0} \phi\right)^{2 p}}{2\left(\sigma /\left(\frac{\nu R_{e}}{l_{0}}\right)\right)^{2}}\right) .
\end{aligned}
$$

Cuando el índice de dimensiones tiende a 1 , debe tenerse que: $2 p \rightarrow 2, p-1 \rightarrow 0$, luego una posible escogencia es: $p=\frac{1}{2}(3-\beta), p-1=\frac{1}{2}(1-\beta), \sigma=\frac{\nu R_{e}}{l_{0}}$. Luego si $v_{0}=\left(\varepsilon l_{0}\right)^{1 / 3}$ satisface una gaussiana $G$, entonces el gradiente $\phi$ satisface la distribución $F, F(\phi)=G\left(v_{0}\right)\left|\frac{d v_{0}}{d \phi}\right|$, y con la normalización $F(\phi=0)=1$, se obtiene,

$$
\begin{aligned}
F(\phi)= & \left(\phi /\left(2\left(\frac{\nu R_{e}}{l_{0}}\right)^{2}\right)^{\frac{1}{3-\beta}}\right)^{\frac{1}{2}(1-\beta)} \exp \left(-\left(\frac{1}{2\left(\frac{\nu R_{e}}{l_{0}}\right)^{2}}\right) \phi^{3-\beta}\right) \\
& \stackrel{\beta \rightarrow 1}{\longrightarrow} \exp \left(-\frac{1}{2}\left(\frac{\phi}{\frac{\nu R_{e}}{l_{0}}}\right)^{2}\right) .
\end{aligned}
$$

Y, entonces, la distribución de los gradientes se reduce a la gaussiana para pequeños números de Reynolds. Representamos en forma gráfica este comportamiento de suerte que para valores del índice de dimensiones de $1 / 3$ y $1 / 7$, se tiene la curva continua negra y la de círculos azules; en tanto que para números de Reynolds 10 veces más grandes se obtiene la curva de cruces cafés de la figura 5. 
Las propiedades más importantes de las distribuciones de los gradientes de las velocidades se destacan en la figura 5. Éstas son, la asimetría, que se observa en las colas más gruesas a la derecha que a la izquierda, que se mide a través del tercer momento central, y se caracteriza por una asimetría positiva; y el aplanamiento de las curvas de distribución cerca de la media, o los incrementos de los radios de curvatura, medida por el cuarto momento central, o curtosis.

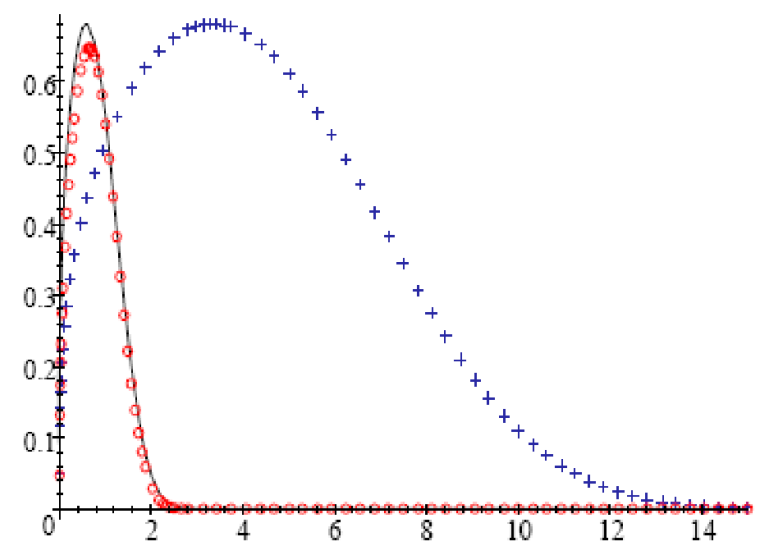

Figura 5: Densidades de los gradientes.

\section{$5 \quad$ Exponentes característicos}

Se puede estimar el número de los exponentes característicos en el volumen del dominio que ocupa la turbulencia desarrollada, tomando en cuenta el fenómeno de la intermitencia, la que se mide por la fracción de llenado del espacio. En determinado rango, el número de exponentes característicos, $N_{c}$, se identifica con el número de valores propios de la parte hermítica del operador de evolución, [20], [14]. Si $\eta$ denota la escala de disipación en donde termina el subrango inercial, el número de los exponentes característicos se estima por la ecuación (40); en donde $n$ señala la etapa del proceso de fractalización; $\Delta t_{n}$, el intervalo de tiempo de esa etapa; $\varepsilon$, la tasa de energía transferida por etapa; y se observa que con la ausencia de la intermitencia, la concentración de exponentes se reduce al cubo del inverso de la escala disipativa, lo cual está en concordancia con la teoría de Kolmogorov.

$$
\left(\frac{N_{h}(\alpha)}{h^{D} T}\right)^{n}=\left(\frac{\varepsilon \Delta t_{n}^{3}}{l_{0}^{2}}\right)^{\frac{1-\beta(\alpha)}{2 / D_{T}+1-\beta(\alpha)}}, \quad N_{c} \approx\left(\frac{N_{h}(\alpha)}{h^{D} T}\right)^{n}\left(\frac{l_{0}}{\eta}\right)^{3} .
$$

\section{Conclusiones}

El objetivo principal de la modelación de la turbulencia radica en superar las características de la distribución gaussiana del modelo de Kolmogorov, pero basados en una teoría asintótica. Las vías, que presentamos en el presente trabajo, son: 
- Suponer que la distribución de la variable aleatoria se concibe como límite de una sucesión de variables aleatorias estacionarias, por lo que surge el modelo de extremos para el número de rasgos que produce la descripción multifractal, la cual depende del índice Lévy y del índice de dimensiones.

- Los procesos potenciados de Bernoulli, vistos como sistema de distribución de energía cinética, nos producen la descripción multifractal que depende de una función de estructura, que se construye a partir de los valores propios de un operador energético; y, para el caso de dos valores propios, uno produce la probabilidad de éxito y el otro la de fracaso, con lo que obtenemos una distribución multifractal acampanada y leptocúrtica, junto con una distribución de las singularidades de tipo sigmoide; y en una forma análoga, también, para el modelo paramagnético.

- Considerando la posibilidad de fracaso en la transmisión de la energía, generalizamos el árbol o cascada energética; luego, a través de un proceso de Cantor generalizado, encontramos la distribución Beta y la función de correlación como su transformada de Laplace, con parámetros de forma que se obtienen de los índices de dimensiones y Lévy.

- Con el sistema de Pearson, reencontramos la distribución Beta con parámetros de forma determinados por la asimetría y la curtosis, los que a su vez dependen de los índices Lévy y de dimensiones.

- La distribución para los gradientes la obtenemos de la distribución de las diferencias de la velocidad y observamos que presentan un incremento de sus colas cuando el número de Reynolds crece.

- La dependencia del índice de dimensiones, nos conduce a plantearnos la siguiente pregunta: ¿para qué valores del índice de dimensiones la forma bilineal, inducida por la velocidad, será coerciva? Nosotros creemos que este valor debe ser cercano a la unidad.

\section{Referencias}

[1] Andreev, A.; Kanto A.; Malo P. (2005) "Simple Approach for Distribution Selection in the Pearson System", Helsingin Kauppakorkeakoulu, Helsinki Schools of Economics, Working Papers, W-388.

[2] Benzi, R.; Biferale, L.; Paladin, G.; Vulpiani, A.; Vergassola, M. (1991) "Multifractality in the statistics of the velocity gradients in turbulence", Phys. Rew. Lett. 67(17): 2299-2302.

[3] Biferale, L. (1993) "Probability distribution functions in turbulent flows and shell models", Phys. Fluids A 5(2): 428-435.

[4] Chorin, A.J. (1994) Vorticity and Turbulence. Springer, New York.

[5] Dressler, R. F. (1953) "Mathematical solution of the problem of roll-waves in inclined open channels". Commu. on Pure and Appl. Math. 6: 149-194. 
[6] Falconer, K. (1990) Fractal Geometry. John Wiley \& Sons, Chichester.

[7] Fefferman, C.L. (2000) "Existence and smoothness of the Navier-Stokes equation". Princeton University, Department of Mathematics, Princeton, May 1.

[8] Halsey, T.C.; Jensen, M.H.; Kadanoff, L. P.; Procaccia, I.; Shraiman, B.I. (1986) "Fractal measures and their singularities: the characterization of strange sets", Phys. Rev A. 33: $1141-1151$.

[9] Hernández, A. (2005) Distribución Límite de los Extremos del Modelo T-student Truncado para Datos de Lluvia Diaria. Tesis de Doctorado, Univ. Simón Bolívar, Caracas.

[10] Koroliuk, V.S. (1981) Manual de la Teoría de Probabilidades y Estadística Matemática. Ed. Mir, Moscú.

[11] Hopf, E. (1950) "The partial differential equation $u_{t}+u \cdot u_{x}=u_{x x}$ ", Comm. Pure and Appl. Math. 3: 201-230.

[12] Lax, P.D. (1968) "Integrals of nonlinear equations of evolution and solitary waves", Comn. Pure and Appl. Math. 21: 467-490.

[13] Levi, E. (1989) El Agua según la Ciencia. Conacyt, Ed. Castell Mexicana, México.

[14] Lieb, E.H. (1984) "On characteristic exponents in turbulence", Commun. Math. Phys. 92: $473-480$.

[15] Meneveau, C.; Sreenivasan, K. (1987) "Simple multifractal cascade model for fully developted turbulence", Phys. Rev. Lett. 59(13): 1424-1427.

[16] Mercado, J.R.; Aldama, Á.A.; Brambila, F. (2004) "Sobre las soluciones de la ecuación de Navier-Stokes", XVIII Congreso Nacional de Hidráulica, San Luis Potosí, SLP, 10 al 12 de nov. de 2004.

[17] Mercado, J.R.; Brambila, F. (2001) "Problemas inversos en las ecuaciones de Fokker-Planck", Aportaciones Matemáticas, Serie Comunicaciones 29, pp. 201-222.

[18] Panton, R.L. (1984) Incompressible Flow. John Wiley \& Sons, New York.

[19] Rose, H A.; Sulem, P.L. (1978) "Fully developed turbulence and statistical mechanics", J. Physique 39: 441-484.

[20] Ruelle, D. (1982) "Large volume limit of the distribution of characteristic exponents in turbulence", Commun. Math. Phys. 87: 287-302.

[21] Scheffer, V. (1977) "Hausdorff measure and the Navier-Stokes equations", Commun. Math. Phys. 55: 97-112.

[22] Smoller, J. (1994) Shock Waves and Reaction-Diffusion Equations. Springer-Verlag, New York.

[23] Tarasov, V.E. (2005) "Fractional Fokker-Planck equation for fractal media", Chaos 15.

[24] Temam, R. (1984) Navier-Stokes Equations. Theory and Numerical Analysis. North-Holland, Amsterdam.

[25] Waymire, E.C. (2005) "Probability \& incomprensible Navier-Stokes equations: An overview of some recent developments", Probability Surveys 2: 1-32. 